\title{
Proper Regulation of Cyclic AMP-Dependent Protein Kinase Is Required for Growth, Conidiation, and Appressorium Function in the Anthracnose Fungus Colletotrichum lagenarium
}

\author{
Yoshitaka Takano, Kenichi Komeda, Kaihei Kojima, and Tetsuro Okuno \\ Laboratory of Plant Pathology, Graduate School of Agriculture, Kyoto University, Kyoto 606-8502, Japan \\ Submitted 13 March 2001; Accepted 5 July 2001.
}

\begin{abstract}
Colletotrichum lagenarium, the casual agent of anthracnose of cucumber, forms specialized infection structures, called appressoria, during infection. To evaluate the role of cAMP signaling in $C$. lagenarium, we isolated and functionally characterized the regulatory subunit gene of the cAMP-dependent protein kinase (PKA). The RPK1 gene encoding the PKA regulatory subunit was isolated from $C$. lagenarium by polymerase chain reaction-based screening. rpk1 mutants, generated by gene replacement, exhibited high PKA activity during vegetative growth, whereas the wild-type strain had basal level activity. The rpk1 mutants showed significant reduction in vegetative growth and conidiation. Furthermore, the rpk1 mutants were nonpathogenic on cucumber plants, whereas they formed lesions when inoculated through wounds. A suppressor mutant showing restored growth and conidiation was isolated from a rpk1 mutant culture. The rpk1-suppressor mutant did not show high PKA activity, unlike the parental rpk1 mutant, suggesting that high PKA activity inhibits normal growth and conidiation. The suppressor mutant, however, was nonpathogenic on cucumber and failed to form lesions, even when inoculated through wounds. The rpk1 and suppressor mutants formed melanized appressoria on the host leaf surface but were unable to generate penetration hyphae. These results suggest that proper regulation of the PKA activity by the $R P K 1$-encoded regulatory subunit is required for growth, conidiation, and appressorium function in C. lagenarium.
\end{abstract}

Colletotrichum species cause destructive anthracnose diseases on numerous crops and ornamental plants worldwide. Symptoms of anthracnose appear on the foliage, stems, or fruits as dark spots or slightly sunken lesions (Agrios 1988). Colletotrichum lagenarium is the causal agent of cucumber anthracnose. Conidia of $C$. lagenarium infect the host plant by forming a specialized infection structure, called an appressorium. Appressoria are swollen, dome-shaped cells that contain melanin layers that are essential for appressorium-mediated

Corresponding author: Y. Takano; E-mail: ytakano@kais.kyoto-u.ac.jp

Nucleotide and amino acid sequence data are available in the DDBJ/EMBL/GenBank databases under accession no. AF353397. penetration (Kubo and Furusawa 1991). In C. lagenarium, three melanin biosynthesis genes ( $P K S 1, S C D 1$, and THRl) required for fungal infection were identified and characterized (Kubo et al. 1996; Perpetua et al. 1996; Takano et al. 1995; Takano et al. 1997b). Magnaporthe grisea, the causal agent of rice blast, forms melanized appressoria that are similar to those formed by Colletotrichum species. It has been suggested that melanized appressoria in M. grisea and Colletotrichum graminicola produce high turgor pressure that enables mechanical penetration into the plants (Bechinger et al. 1999; de Jong et al. 1997; Howard et al. 1991). Dissection of the molecular basis of appressorium formation and penetration-related processes is under intense investigation. Molecular genetic analysis has demonstrated that several signal transduction pathways are involved in the appressorium-mediated infection processes of $M$. grisea and Colletotrichum species. In $M$. grisea, two mitogen-activated protein kinase (MAPK) genes, PMK1 and MPS1, have been shown to be required for the fungal infection process (Xu and Hamer 1996; Xu et al. 1998). In $C$. lagenarium, the CMK1 MAPK gene was shown to regulate conidial germination, appressorium formation, and invasive growth in plants (Takano et al. 2000). Recently, a MAPK kinase (MEK) gene of $C$. gloeosporioides was shown to be involved in multiple steps of fungal pathogenesis, including appressorium formation (Kim et al. 2000).

The cyclic AMP (cAMP) signaling pathway also has been shown to play pivotal roles for fungal pathogenesis (Kronstad 1997; Kronstad et al. 1998). cAMP is produced from ATP by adenylate cyclase and acts as a secondary messenger. In eukaryotic cells, cAMP controls the activity of the cAMPdependent protein kinase (PKA) (Taylor et al. 1990). PKA is a tetrameric holoenzyme that is composed of two regulatory subunits and two catalytic subunits. The holoenzyme tetramer is inactive and unable to phosphorylate substrate proteins. Binding cAMP to regulatory subunits releases the catalytic subunits, enabling phosphorylation of target proteins involved in cAMP-regulated processes. Fungi employ cAMP signaling to regulate a variety of processes. In Saccharomyces cerevisiae, cAMP signaling pathways regulate resistance to environmental stress, growth on many carbon sources, glycogen accumulation, cell cycle progression, sporulation, and pseudohyphal growth (Cameron et al. 1988; Pan and Heitman 1999; Robertson and Fink 1998; Toda et al. 1987; Tokiwa et al. 1994). In the phytopathogenic fungus Ustilago maydis, cAMP 
signaling was shown to be involved in the morphological switch between budding and filamentous growth and in fungal pathogenesis, including gall formation (Gold et al. 1994; Gold et al. 1997). In M. grisea, several genes involved in the cAMP pathway have been functionally characterized. The disruption of the $M$. grisea adenylate cyclase gene $M A C 1$ resulted in pleiotropic defects on growth, conidiation, sexual development, and appressorium formation, indicating that the cAMP signaling pathway regulates multiple aspects, including pathogenicity, in this fungus (Adachi and Hamer 1998; Choi and Dean 1997). In addition, it was reported that a point mutation of the PKA regulatory subunit gene (SUM1) partially restored the defects of the macl mutant (Adachi and Hamer 1998). In contrast to the macl mutants, mutants disrupted in the catalytic subunit gene of PKA $(C P K A)$ exhibited normal growth and conidiation but showed loss of pathogenicity, which resulted from the defect in formation of functional appressoria (Mitchell and Dean 1995; Xu et al. 1997). The catalytic subunit gene of PKA in Colletotrichum trifolii has been shown to be necessary for penetration by appressoria, which is similar to the phenotype of the cpkA mutants in M. grisea (Yang and Dickman 1999a). In addition, the regulatory subunit gene of PKA was isolated recently from $C$. trifolii and shown to complement the Neurospora crassa mcb mutant carrying a temperature-sensitive mutation in the regulatory subunit gene of PKA (Yang and Dickman 1999b). The roles of this gene in $C$. trifolii, however, remain to be elucidated.

In this report, we isolated the RPKl gene encoding the regulatory subunit of PKA from $C$. lagenarium. The RPK1deleted strains had severe defects in growth and conidiation. The rpkl mutants showed attenuated pathogenicity to cucumber. From the rpkl mutant, we also isolated a suppressor mutant, showing growth and conidiation similar to those of the wild type. Further phenotypic and biochemical analyses of the rpkl and rpkl-suppressor mutants suggest that proper regulation of the cAMP-PKA signaling pathway is necessary for pathogenesis and development in $C$. lagenarium.

\section{RESULTS}

\section{Isolation of $C$. lagenarium RPK1 gene.}

A polymerase chain reaction (PCR)-based screen with degenerate oligonucleotide primers (see below) was used to iso-
A

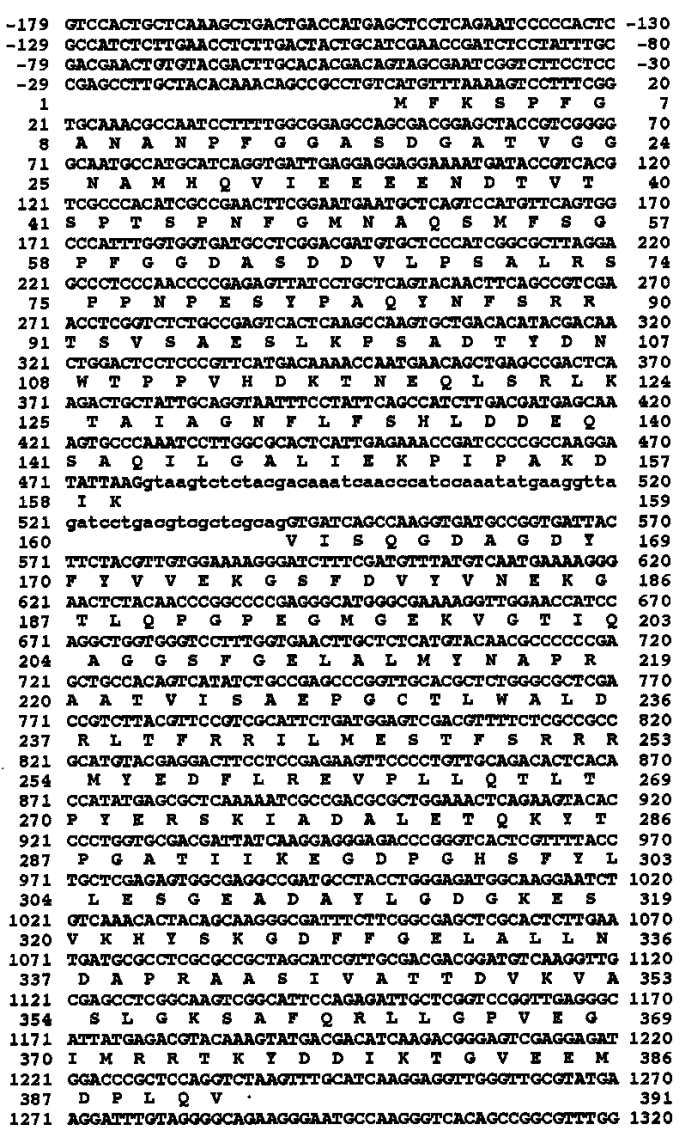

B

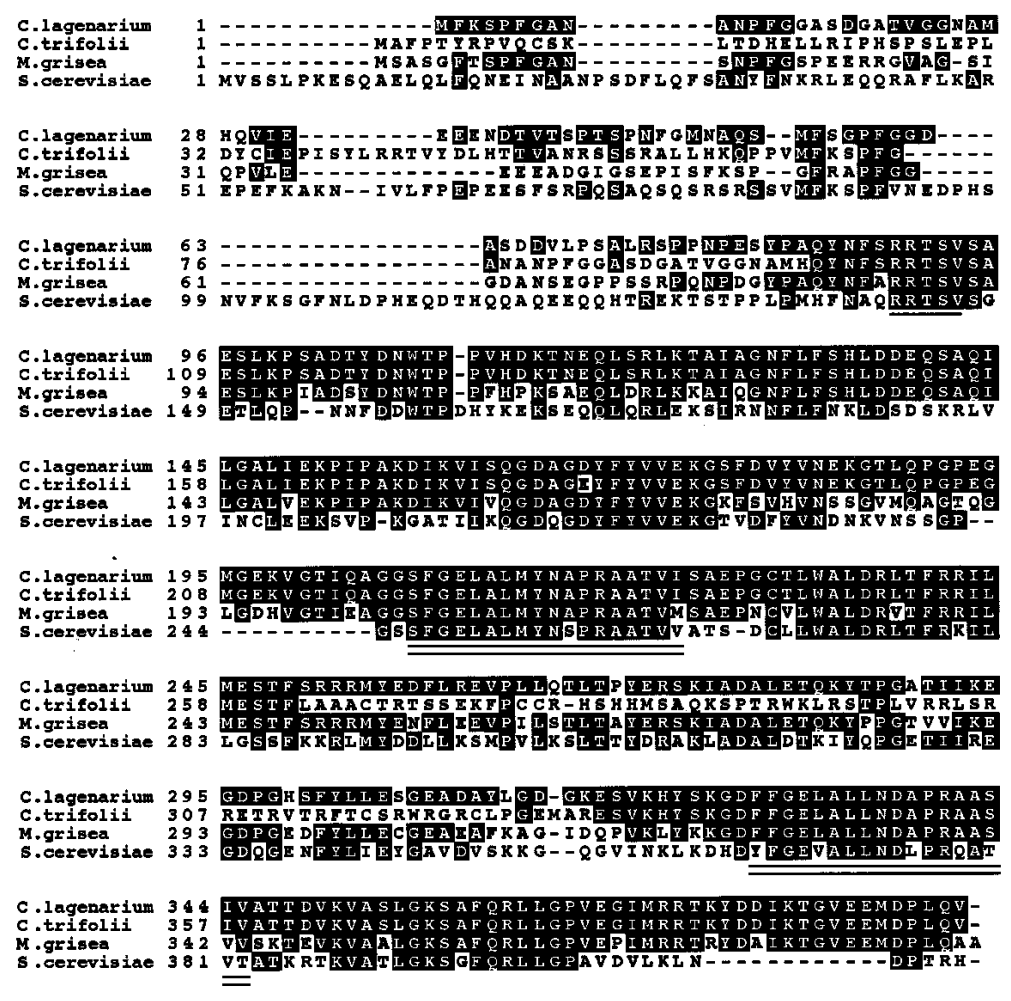

Fig. 1. The Colletotrichum lagenarium RPK1 gene encoding the regulatory subunit of protein kinase (PKA). A, Nucleotide and deduced amino acid sequences of $R P K 1$. Intron sequences are indicated in lowercase. DDBJ/EMBL/GenBank accession no. for RPK1 is AF353397. B, Amino acid sequence alignment of Rpk1 in C. lagenarium with those of the regulatory subunit of PKA in other organisms. Deduced amino acid sequence of Rpk1 was aligned with those of Ct-Pkar in Colletotrichum trifolii, Sum1 in Magnaporthe grisea, and Bcy1 in Saccharomyces cerevisiae. Sequences were aligned with the CLUSTAL W program (Thompson et al. 1994). Identical amino acids are indicated as white letters on a black background. Similar residues are shown on a gray background. Gaps introduced for the alignment are indicated by hyphens. The kinase inhibition domain is underlined. Cyclic AMP binding domains are double underlined. DDBJ/EMBL/GenBank accession nos. for Ct-Pkar, Sum1, and Bcy1 are AF046922, AF024633, and M15756, respectively. 
late a gene encoding a regulatory subunit of PKA from $C$. lagenarium. A cDNA pool synthesized from mRNA of conidia during appressorium formation (at $2 \mathrm{~h}$ postincubation) was used as a template for PCR screening (Inagaki et al. 2000). Amplified $0.8-\mathrm{kb}$ PCR products were cloned into pBluescript and sequenced. The insert sequence of clone pRPK1 showed a high degree of identity with the regulatory subunit genes of PKA in other organisms. This insert was used to probe for and isolate genomic cosmid clones containing the regulatory subunit gene of PKA, designated $R P K 1$. The entire nucleotide sequence of the RPKI gene was determined. $R P K 1$ contained a 1,239-bp open reading frame encoding a 391 amino acid protein with one intron (Fig. 1A). The intron region was confirmed by analysis of reverse-transcription (RT)-PCR products of the RPKl transcript (data not shown). The deduced amino acid sequence of $R P K 1$ shared significant homology with those of the PKA regulatory subunit genes: $M$. grisea SUM1 (71\% identity), N. crassa MCB (70\%), C. trifolii Ct-PKAR (69\%), and Saccharomyces cerevisiae BCY1 (40\%) (Fig. 1B). Rpk1 contained a predicted conserved kinase inhibition site and two cAMP binding domains (Fig. 1B). These results strongly suggest that the $R P K l$ gene encodes the regulatory subunit of PKA in $C$. lagenarium. DNA gel blot analysis with genomic DNAs digested with BamHI, EcoRI, and PstI indi-

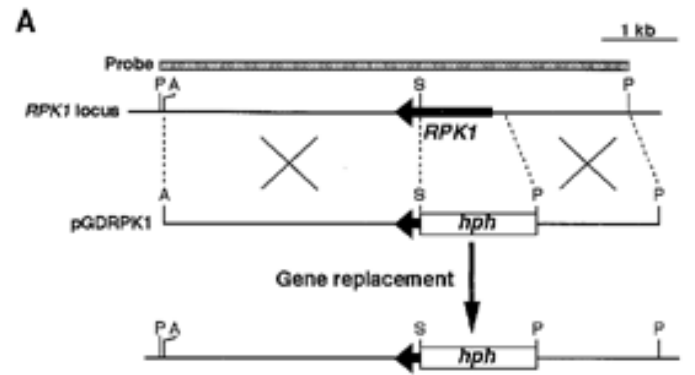

B

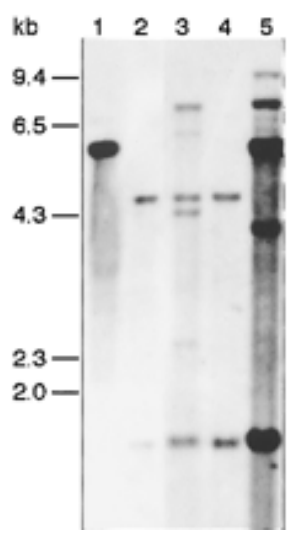

Fig. 2. Gene disruption of $R P K 1$. A, $R P K 1$ locus and gene-replacement vector pGDRPK1, which contains a hygromycin-resistant gene $(h p h)$ cassette flanked by border sequences of RPK1. By homologous recombination through double crossing, the major region of $R P K 1$ was replaced by the hph cassette. A, ApaI; P, PstI; S, SmaI. B, DNA gel blot analysis of the RPK1 gene-replacement transformants. Genomic DNAs were extracted from the wild-type strain 104-T (lane 1); RPK1 disrupted transformants DPK1, DPK3, and DPK4 (lanes 2 to 4); and ectopic transformant DPK2 (lane 5). All DNA samples were digested with PstI. Blot was probed with the 6.1-kb PstI fragment, indicated by a gray bar in $\mathbf{A}$ cates that $R P K 1$ is a single-copy gene in the $C$. lagenarium wild-type strain (data not shown).

\section{Inactivation of $R P K 1$ caused reduction of growth and conidiation.}

To investigate the roles of $R P K 1$ in $C$. lagenarium, we generated $R P K 1$ knockout mutants by one-step gene replacement. The gene-disruption vector pGDRPK1 containing a hygromycin phosphotransferase gene $(h p h)$ and the $5^{\prime}$ and $3^{\prime}$ flanking regions of $R P K 1$ was constructed (Fig. 2A). pGDRPK1 was introduced into wild-type strain 104-T, and transformants were selected on medium containing hygromycin. Among 36 hygromycin-resistant transformants, 23 exhibited reduced growth compared with the wild-type strain and four were subjected to DNA gel blot analysis to investigate the gene-replacement events of RPK1 (Fig. 2B). Genomic DNA was digested with $P s t \mathrm{I}$ and probed with a 6.1-kb PstI fragment containing RPK1. Transformant DPK2 showing the wild-type phenotype contained the 6.1-kb PstI fragment common to wild-type 104-T. DPK2 also contained one additional band, indicating that pGDPKAR was introduced through ectopic integration. Transformants DPK1, DPK3, and DPK4, however, showed a defect in growth and lost the 6.1-kb PstI fragment, yet contained common 4.8- and 1.6-kb PstI fragments, which is consistent with the expected length for the gene replacement event (Fig. $2 \mathrm{~A}$ and $\mathrm{B}$ ). Additional fragments were detected in transformant DPK3, suggesting that the plasmid also may be introduced through ectopic integration. We conclude that RPKI was disrupted in these three transformants. Strains DPK1 and DPK4 were subjected to further analysis.

The rpkl $\Delta$ strains DPK1 and DPK4 showed significant reduction in mycelial growth on potato dextrose agar (PDA)

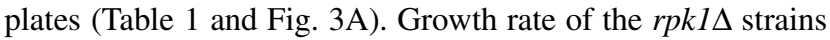
was approximately $60 \%$ of that in the wild type. Melanization of mycelia of these mutants on PDA was decreased compared with the wild type (Fig. 3A). Conidial production also was reduced significantly in the rpkl $1 \Delta$ strains (Table 1). The wildtype strain produced an average of $23.6 \times 10^{6}$ conidia per culture plate. The ectopic transformant DPK2 produced similar numbers of conidia as the wild type. The $r p k 1 \Delta$ strains produced less than $10^{5}$ conidia per plate. Some conidia (5 to $10 \%$ ) from the $r p k 1 \Delta$ strains formed germ tube-like structures on mycelial cultures grown on PDA (Fig. 4), whereas conidia of the wild-type fungal cultures did not form those structures (data not shown). The $r p k 1 \Delta$ strains also showed a significant

Table 1. Characteristics of $r p k 1$ mutants and the suppressor mutant

\begin{tabular}{lccc}
\hline Strain $^{\mathbf{a}}$ & $\boldsymbol{R P K 1}$ & ${\text { Vegetative growth }(\mathbf{m m})^{\mathbf{b}}}^{\text {Conidia }\left(\times \mathbf{1 0}^{\mathbf{5}}\right)^{\mathbf{c}}}$ \\
\hline 104-T & + & $32.0 \pm 1.3$ & $236.6 \pm 90.7$ \\
DPK1 & - & $18.3 \pm 2.2$ & $0.7 \pm 0.21$ \\
DPK4 & - & $18.5 \pm 3.5$ & $0.4 \pm 0.31$ \\
DPK2 & + & $32.0 \pm 1.6$ & $286.6 \pm 20.8$ \\
SGC1 & - & $29.6 \pm 1.4$ & $198.3 \pm 43.6$ \\
\hline
\end{tabular}

a $104-\mathrm{T}$ is the wild-type strain. DPK1 and DPK4 are the rpk1 mutants. DPK2 is an ectopic integration transformant. SGC1 is the suppressor mutant of DPK1.

${ }^{\mathrm{b}}$ Strains were grown on potato dextrose agar at $24^{\circ} \mathrm{C}$ for 7 days and colony diameter was measured. Each value represents mean \pm standard deviation from three independent experiments.

${ }^{\mathrm{c}}$ Conidia were collected from 7-day-old cultures and counted. Each value represents mean \pm standard deviation from three independent experiments. 
reduction in growth and conidiation on minimal medium agar plates compared with the wild type (data not shown). These results demonstrate that $R P K 1$ is involved in vegetative growth and conidiation.

We used the model PKA substrate Kemptide (Promega, Madison, WI, U.S.A.) to assay PKA activity in protein extracts from vegetative mycelia of wild-type strain 104-T, ectopic transformant DPK2, and $r p k 1 \Delta$ strains DPK1 and DPK4 (Fig. 3B). In mycelia of the wild-type and ectopic transformant, PKA activity was undetectable. The addition of cAMP into protein extracts of wild-type mycelia elevated PKA activity, indicating that PKA proteins are present as an inactive form in vegetative mycelia. In contrast, the $r p k 1 \Delta$ strains exhibited high PKA activity in the absence of cAMP, whereas the addition of cAMP did not elevate PKA activity in these strains. PKA activity in the rpkl mutants did not reach the level of the wild type in the presence of cAMP. We conclude that the RPK1 gene encodes the regulatory subunit of PKA and that $R P K 1$ controls the growth and conidiation in C. lagenarium via a cAMP-mediated signal transduction pathway.

\section{Suppressor mutant of the $r p k 1 \Delta$ strain lost PKA activity.}

The rpkls strains formed compact gray colonies. During long incubation, however, we occasionally found a growthsuppressor mutant sector on cultures of $r p k 1 \Delta$ strain DPK1 grown on solid medium. A suppressor mutant, designated

\section{A}

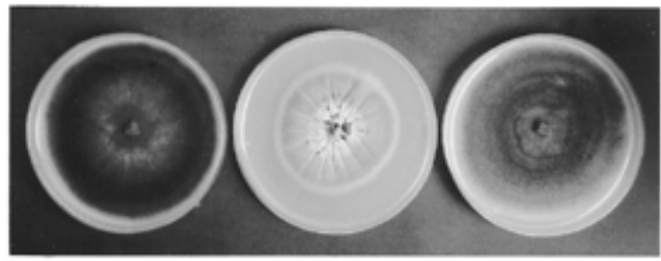

B

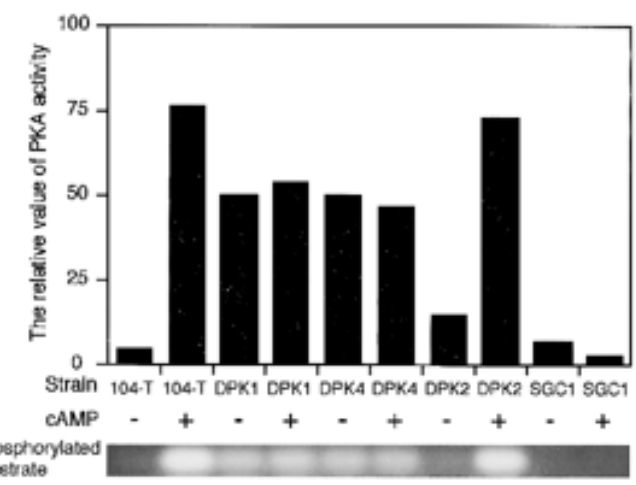

Fig. 3. Mycelial growth and protein kinase (PKA) activity in $r p k 1 \Delta$ strains and suppressor mutant. A, Mycelial growth in wild-type and mutant strains. Wild-type strain 104-T (left), rpk1 mutant DPK1 (center), and suppressor mutant SGC1 (right) were incubated on a potato dextrose agar plate at $24^{\circ} \mathrm{C}$ for 20 days. B, PKA activity in the wild-type and mutant strains. Protein extracts were prepared from the mycelia of wild-type strain 104-T, rpk1 mutants DPK1 and DPK4, ectopic transformant DPK2, and suppressor mutant SGC1. PKA assays were performed with a model substrate (Kemptide; Promega, Madison, WI, U.S.A.), with (+) or without (-) $1 \mu \mathrm{M}$ cyclic AMP. Relative amount of the phosphorylated substrate, separated on agarose gel, was determined with the National Institutes of Health image program, version 1.61 (Bethesda, MD, U.S.A.).
SGC1 (for suppressor for growth and conidiation), was isolated. Strain SGC1 demonstrated a significantly increased growth rate and conidial production compared with the $r p k 1 \Delta$ strain on PDA plates, although growth and conidiation of SGC1 did not reach the wild-type level (Fig. 3A and Table 1). Also, colonies of SGC1 were darkly melanized, which is similar to the wild type but unlike the parental $r p k 1 \Delta$ strain DPK1 (Fig. 3A). To determine whether SGC1 retained the high PKA activity shown in rpkl $1 \Delta$ strain DPK1, we measured the PKA activity in SGC1 during mycelial growth (Fig. 3B). In the absence of cAMP, the PKA assay revealed that SGC1 exhibited similarly low PKA activity as the wild type. This result demonstrates that a mutation reducing constitutive PKA activity in the rpk1 mutant occurred in SGC1. In contrast to the wild type, the addition of cAMP did not elevate PKA activity in SGC1. This result strongly suggests that SGC1 lost a majority of functional PKA activity. From these results, we conclude that loss of constitutively high PKA activity permits growth and conidiation in SGC1 to levels similar to those in the wildtype strain and that high PKA activity impairs these aspects of development.

\section{RPK1 is required for fungal pathogenicity.}

To assess the pathogenicity of $r p k 1 \Delta$ strains, inoculation assays were performed on host cucumber leaves. Twenty-microliter drops of conidial suspension from strains of the wild

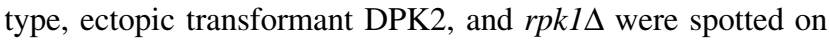
detached cucumber leaves and incubated. The wild type formed lesions that first appeared yellowish, then turned brown (Fig. 5). On leaves inoculated with ectopic transformant DPK2, wild-type lesions were found (data not shown). In contrast, $r p k 1 \Delta$ strain DPK1 showed significant reduction of pathogenicity because it formed no detectable lesions or formed only tiny yellow spots (Fig. 5A). Similar results were observed for $r p k 1 \Delta$ strain DPK4 (data not shown). We also performed spray-inoculation assays of conidial suspension on cucumber leaves. In these assays, $r p k 1 \Delta$ strain DPK1 formed few lesions on cucumber leaves, whereas the wild-type strain formed many (data not shown). These results indicate that the $R P K 1$ gene is necessary for fungal pathogenicity in C. lagenarium. We also investigated the pathogenicity of the suppressor mutant SGC1, which is derived from $r p k 1 \Delta$ strain DPK1. Remarkably, strain SGC1 did not form any lesions on cucum-
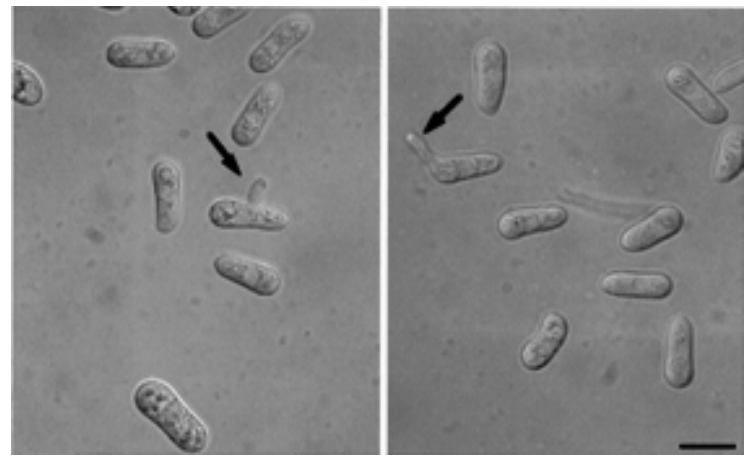

Fig. 4. Misscheduled germination of the rpkl mutant conidia on mycelial cultures. A portion of the conidia in the $r p k 1$ mutants germinated irregularly on mycelial culture grown on potato dextrose agar plates. Germinating conidia of the rpkl mutant in both panels are indicated by arrows. $\mathrm{Bar}=10 \mu \mathrm{m}$ 
ber leaves in a drop- or spray-inoculation assay (Fig. 5B and data not shown). This suggests that a mutation occurred in SGC1 that restored defects in growth and conidiation of the rpkl mutant but did not restore defects in pathogenicity.

\section{Phenotypic analysis of the rpk1 and its suppressor mutants during the infection process.}

To determine the steps leading to deficiency in the pathogenicity of the rpkl mutants, the infection-related morphogenesis of $r p k 1 \Delta$ strain DPK1 was investigated (Fig. 6). DPK1 germinated and formed darkly melanized appressoria on a glass surface (data not shown). DPK1 also formed appressoria on the host leaf tissue at $12 \mathrm{~h}$ postinoculation (Fig. 6B). Appressoria of the wild type formed penetration hyphae on cucumber leaves at 3 to 4 days postinoculation (Fig. 6D). In contrast, appressoria of DPK1 did not form penetration hy-

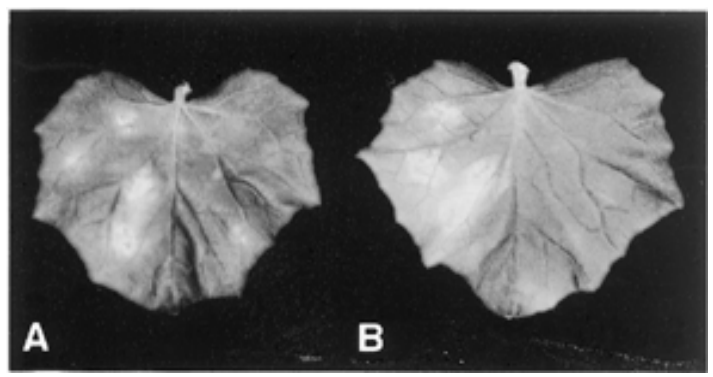

Fig. 5. Infection assays on cucumber leaves. Detached cucumber leaves were drop-inoculated and incubated at $24^{\circ} \mathrm{C}$ for 7 days. On the left half of the leaves, drops of conidial suspension of wild-type 104-T were placed as a positive control. On the right half of the leaves, A, conidial suspensions of rpk1 mutant DPK1 and B, suppressor mutant SGC1 were spotted. phae on host tissues (Fig. 6E). Similar results were obtained for $r p k 1 \Delta$ strain DPK4 (data not shown), suggesting that loss of pathogenicity is the result of a defect in appressoriummediated penetration and that $R P K l$ is required for normal appressorium function.

We investigated appressorium formation of suppressor strain SGC1. On the host plant, SGC1 germinated and formed appressoria at $12 \mathrm{~h}$ postinoculation (Fig. 6C). Appressoria formed by SGC1 were as darkly melanized as those formed by the wild type, although they could not form penetration hyphae into the host plants (Fig. 6F). This result suggests that loss of pathogenicity in SGC1 is the result of a deficiency in the ability to develop penetration hyphae. Interestingly, SGC1 failed to germinate efficiently on a glass surface. At $12 \mathrm{~h}$ postincubation on glass petri dishes, less than $30 \%$ of SGC1 conidia germinated, whereas $>90 \%$ of conidia from the wild-type strain germinated. At $48 \mathrm{~h}$ postincubation, the germination rate of SGC1 was increased slightly but still not over $50 \%$. Germinating conidia of SGC1 formed melanized appressoria on the glass surface as well as on the host surface (data not shown).

We investigated the ability of the rpk1 mutant DPK1 and its suppressor mutant SGC1 to grow invasively in plants by inoculation assay through wounded sites of cucumber leaves (Fig. 7). We used albino mutant 79215, which carries a mutation of the melanin gene PKSI and cannot penetrate because of a lack of melanization in appressoria, as a positive control strain retaining invasive growth ability. As a result, rpkl mutant DPK1 formed lesions on wounded plants, although the lesions were relatively smaller compared with those formed by the control strain 79215 (pksl) (Fig. 7A and B). This result indicates that DPK1 retained invasive growth ability in cucumber leaves. In contrast, SGC1 did not develop lesions but, rather, tiny, yellowish or brown dots similar to those formed
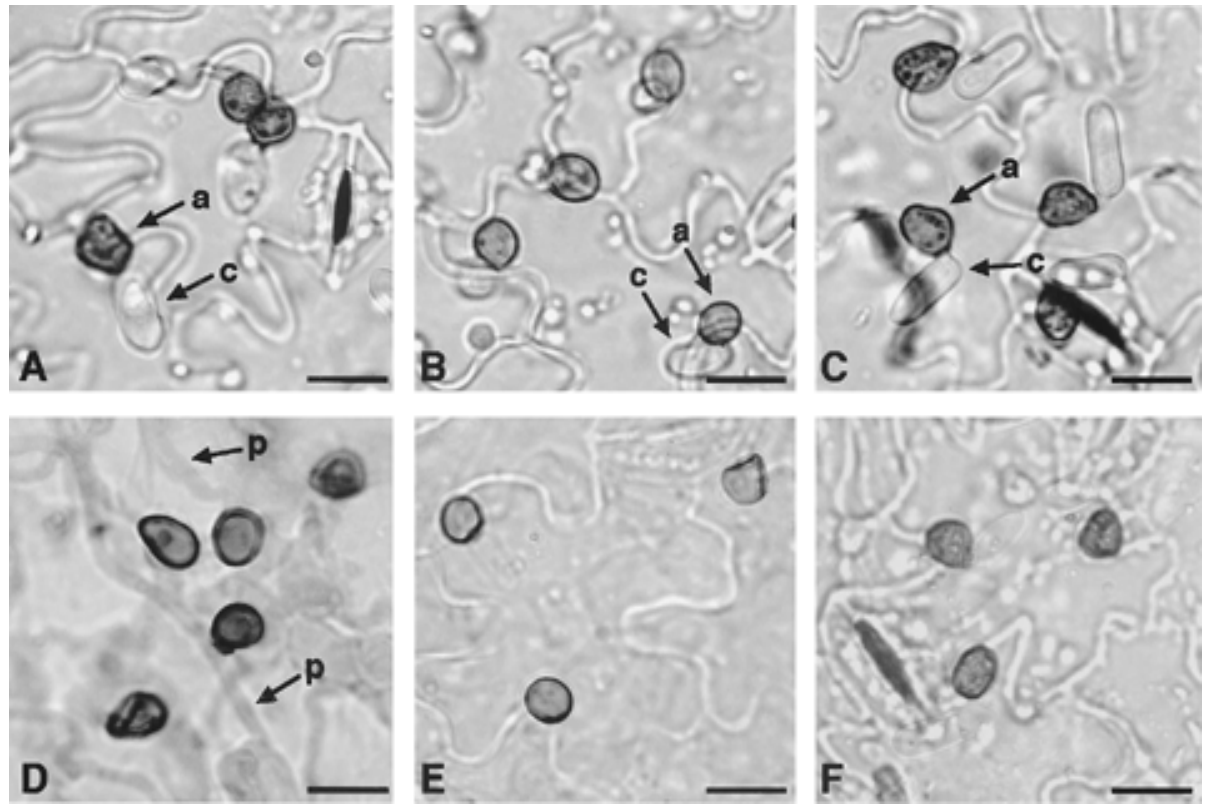

Fig. 6. Appressorium formation and penetration of the wild type and mutant strains on cucumber leaves. Drops of conidial suspensions of wild-type strain 104T, rpk1 mutant DPK1, and suppressor mutant SGC1 were spotted on cucumber cotyledons. A-C, Germination and appressorium formation. Inoculated leaves were incubated at $24^{\circ} \mathrm{C}$ for $12 \mathrm{~h}$. D-F, Formation of penetration hyphae by appressoria. Leaves were incubated at $24^{\circ} \mathrm{C}$ for 4 days. A and D, 104-T. B and E, DPK1. C and F, SGC1. Appressoria of strains DPK1 and SGC1 did not form penetration hyphae, whereas wild type 104-T formed efficiently. a, appressorium; c, conidium; p, penetration hypha. Bar $=10 \mu \mathrm{m}$. 
on wounded sites where water was spotted as a negative control (Fig. 7C). This result suggests that the SGC1 strain was impaired in invasive growth in cucumber leaves.

\section{DISCUSSION}

\section{Roles of PKA for growth and conidiation.}

We isolated and characterized the RPKl gene encoding the regulatory subunit of PKA in $C$. lagenarium. The deduced amino acid sequence of RPK1 showed highest homology (71\%) to that of SUMI in $M$. grisea. In addition, C. lagenarium RPKI and M. grisea SUMI only have one intron, which is located in the same position, in reference to flanking amino acid sequences (KDIK and VI(S/V)Q), whereas $C t$-PKAR of $C$. trifolii, the fungus closely related to $C$. lagenarium, has $69 \%$ homology to $C$. lagenarium RPK1 and contains two in-

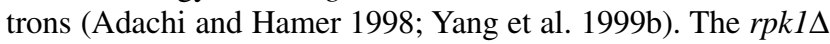
strains, generated by gene replacement, showed a severe reduction in growth rate and conidiation on nutrient agar, indicating that RPKI is required for these developments in $C$. lagenarium. PKA assays demonstrated that the $r p k l$ mutants, unlike the wild type, have high PKA activity during vegetative growth. These results suggest that improper high PKA activity inhibits normal growth and conidiation in rpkl mutants. In $M$. grisea, a mutation in SUM1 (sum1-99) suppresses defects in the growth and conidiation of the macl mutant that lacks the adenylate cyclase (Adachi and Hamer 1998). The $\Delta m a c 1$ sum1-99 mutant of $M$. grisea showed high PKA activity during mycelial growth in the absence of cAMP, implying that increased PKA activity did not inhibit fungal growth and conidiation in $M$. grisea. In the $\Delta$ macl suml-99 mutant, however, PKA activity was partially stimulated by the addition of cAMP, whereas cAMP did not elevate PKA activity in $C$. lagenarium rpkls strains. This suggests that the regulatory subunit of PKA (Sum1) was not inactivated completely by the sum1-99 mutation. It will be interesting to see whether the deletion of SUMI in M. grisea causes a reduction in growth and conidiation. It has been reported that regulatory subunit genes of PKA regulate morphogenesis of fungal cells in $N$. crassa and $U$. maydis. $U$. maydis $u b c$ mutants that have been disrupted in the PKA regulatory subunit gene exhibited constitutive budding growth and failed gall formation (Gold et al. 1994; Gold et al. 1997). In N. crassa, a temperature-sensitive mutation $(m c b)$ in the regulatory subunit gene severely affected growth polarity at restrictive temperatures (Bruno et al. 1996). Consistent with these reports, some conidia in the rpkl mutants developed germ tube-like structures on mycelial cultures, indicating involvement of $R P K 1$ in conidium maturity or dormancy. This result also suggests that activation of the PKA pathway by disruption of $R P K 1$-induced misscheduled germination of conidia on mycelial colonies.

The finding that the addition of cAMP elevated PKA activity in mycelial extracts of the wild type indicates that a certain amount of PKA catalytic subunits inactivated by Rpk1 is present in the vegetative growth stage. In the presence of cAMP, PKA activity in extracts of mycelia of the $r p k 1$ mutants was lower than that of the wild type, suggesting a possibility that there is a lower amount of catalytic subunits in the mycelia of rpkl mutants than in the wild type. Catalytic subunits released from Rpk1 might be unstable compared with those forming holoenzymes with Rpk1. Strain SGC1, the suppressor mutant of the rpk1 mutant DPK1 showed no detectable PKA activity, even in the presence of cAMP, suggesting that SGC1 lost a majority of functional catalytic subunits. In $S$. cerevisiae, a mutation in the catalytic subunit gene TPK1 suppressed the defects of the $b c y l$ mutant disrupted in the regulatory subunit (Cameron et al. 1988). Recently, we isolated a catalytic subunit gene of PKA from $C$. lagenarium and introduced the genome fragment containing this gene into $\mathrm{SGC1}$. As a result, 16 of 26 SGC1 transformants with the catalytic subunit gene of PKA formed compact and less melanized colonies, similar to those formed by the rpkls strains (N. Takayanagi, K. Kojima, Y. Takano, and T. Okuno, unpublished results). This suggests that a mutation in the catalytic subunit gene of PKA produces the suppressor mutant SGC1 from the $r p k 1 \Delta$ strain. The finding that SGC1 grew efficiently and produced conidia suggests that major PKA activity is dispensable for the growth and conidiation of $C$. lagenarium on nutrient agar media. Consistently, in $C$. trifolii and $M$. grisea, the disruption of PKA catalytic subunit genes did not cause severe effects on either growth or conidiation.

\section{Regulation of PKA during fungal infection.}

Strain SGC1, which lost major PKA activity, lacked pathogenicity on cucumber. The conidia of SGC1 formed wellmelanized appressoria on the host plant, but appressoria did not form penetration hyphae into the host plant. This suggests that PKA activity is necessary for appressorium function in $C$. lagenarium, which is supported by previous reports that mutants of $M$. grisea cpkA and C. trifolii ct-pkac that have been disrupted in catalytic subunit genes of PKA are defective in appressorial penetration (Xu et al. 1997; Yang and Dickman 1999a). An inoculation assay through wounded sites of plants, however, suggests that SGC1 was impaired in invasive growth. This result is not consistent with the phenotypes of $M$. grisea cpkA and $C$. trifolii ct-pkac mutants that retain invasive growth ability. At present, we cannot exclude the possibility that SGC1 possesses a mutation affecting invasive growth, in addition to the mutation impairing PKA activity. The rpkl mutants exhibited severely reduced pathogenicity, whereas they were able to form lesions through the wounded sites of plants. Appressoria formed by the rpkl mutants failed to form penetration hyphae into the host plant. This suggests that constitutive activation of PKA also impaired appressorium function in C. lagenarium. In C. trifolii, PKA activity is developmentally regulated during appressorium formation (Yang and Dickman 1997). Thus, we consider that the proper regulation of PKA activity during appressorium formation and penetration processes is necessary for appressorial penetration in $C$. lagenarium. In the $\Delta$ macl suml-99 mutant of $M$. grisea, conidia from a 6-day-old culture showed similar pathogenicity as in the wild type, whereas conidia from a 15-day-old culture showed reduced pathogenicity, which is thought to be the result of a different degree of glycogen and lipid degradation in the mutant (Thines et al. 2000). In contrast, the conidia of the $C$. lagenarium rpkl mutants from 6-day-old cultures exhibited a significant reduction in pathogenicity. It remains to be seen whether the rpkl mutants exhibit rapid degradation of glycogen and lipid, similar to the $\Delta$ macl suml-99 mutant of $M$. grisea. Strain SGC1 showed a reduced germination ability on a glass surface, whereas the rpkl mutants germinated efficiently. This suggests the possibility that activation of PKA 
promotes germination of conidia on a glass surface. Consistent with our assumption, an involvement of the cAMP-PKA pathways in conidial germination has been implied by the finding that $c t$-pkac mutants of $C$. trifolii display a delayed germination on a glass surface (Yang and Dickman 1999a). On the host plant surface, a germination deficiency in SGC1 was not obvious, suggesting that chemical and/or physical properties on the host plant surface triggered germination in the mutant.

In C. gloeosporioides, host surface wax has been shown to trigger germination and appressorium formation (Podila et al. 1993). It therefore seems plausible that germination of SGC1 is enhanced by a putative germination pathway, which is activated by host surface compounds. We have shown that deletion of $C M K 1$ MAP kinase gene result in reduced germination of conidia on glass and host surfaces (Takano et al. 2000). We thus speculate that MAP kinase and cAMP-PKA pathways regulate conidial germination in $C$. lagenarium. The $\mathrm{cmkl}$ mutants lost pathogenicity because of multiple defects in pathogenesis steps, including germination and appressorium formation. It is evident that the cAMP-PKA and CMK1-encoded MAP kinase pathways are required for fungal pathogenesis in C. lagenarium. Furthermore, we found recently that an additional MAP kinase gene, designated $M A F 1$, regulates appressorium differentiation in $C$. lagenarium, independent of CMK1 (K. Kojima, T. Kikuchi, Y. Takano, E. Oshiro, and T. Okuno, unpublished results). This suggests that multiple signaling pathways are involved in the fungal pathogenesis process of $C$. lagenarium. Additional studies concerning the roles of cAMP-PKA and MAP kinase pathways will enable us to understand how fungal pathogens utilize signaling pathways to establish infection in their host plants and will provide novel targets for the future design of antifungal molecules.

\section{MATERIALS AND METHODS}

\section{Fungal strains and culture conditions.}

C. lagenarium strain 104-T was used as the wild-type strain. C. lagenarium cultures were maintained on PDA (3.9\% [wt/vol]; Difco, Detroit, MI, U.S.A.) at $24^{\circ} \mathrm{C}$ in the dark. In addition to the PDA plates, a minimal medium (MM) agar plate also was used to test growth and conidiation. The MM contained $10 \mathrm{~g}$ of glucose, $1 \mathrm{ml}$ of trace elements, $1 \mathrm{ml}$ of vitamin solution, $6 \mathrm{~g}$ of $\mathrm{NaNO}_{3}, 0.5 \mathrm{~g}$ of $\mathrm{KCl}, 0.5 \mathrm{~g}$ of $\mathrm{MgSO}_{4}$, and $1.5 \mathrm{~g}$ of $\mathrm{KH}_{2} \mathrm{PO}_{4}(\mathrm{pH} 6.5)$, in 1 liter of water (Beckerman and Ebbole 1996).

\section{Cloning of RPK1.}

A PCR-based screening was performed to isolate the $R P K 1$ gene with cDNA synthesized from polyadenylated RNA prepared from conidia at $2 \mathrm{~h}$ postincubation (Inagaki et al. 2000). Primers PKAR-S1 (5'-TACGACAACTGGACTCCNCC-3') and PKAR-AS1 (TCTCTGGAATGCCGACTTNCC) were designed based on the conserved amino acid and nucleotide sequences of the regulatory subunit of PKA in $C$. trifolii, $M$. grisea, and $N$. crassa. The PCR reaction was performed with Ex Taq polymerase (Takara, Ohtsu, Japan). PCR conditions were 30 cycles at $94^{\circ} \mathrm{C}$ for $1 \mathrm{~min}, 52^{\circ} \mathrm{C}$ for $2 \mathrm{~min}, 72^{\circ} \mathrm{C}$ for $2 \mathrm{~min}$, and a final extension at $72^{\circ} \mathrm{C}$ for $5 \mathrm{~min}$. PCR products were introduced into pGEM-T vector (Promega) according to manufacturer's instructions and sequenced. Screening by sequenc- ing identified one clone, pRPK1, exhibiting significant homology with the regulatory subunit of PKA in other organisms. The insert fragment of pRPK1 was used as a probe to screen genomic clones containing the RPKI gene from the C. lagenarium cosmid library, and three independent cosmid clones were isolated. The DNA sequence was determined with the Big Dye terminator cycle sequencing ready reaction kit (Applied Biosystems, Warrington, U.K.) and a Model 310 automated DNA sequencer (Applied Biosystems). To confirm the intron sequence of the $R P K 1$ gene, a RT-PCR experiment was performed.

\section{Construction of gene-replacement vector pGDRPK1.}

pCB1636 (Sweigard et al. 1997) containing the hph gene was digested with XhoI and blunted with T4 DNA polymerase, which was followed by ApaI digestion. The 3.3-kb ApaI-SmaI fragment containing the 3 ' flanking region of RPK1 was ligated with the enzyme-treated pCB1636, described above, and transformed to Escherichia coli to produce plasmid pGDAS3. The $1.6-\mathrm{kb}$ fragment containing the $5^{\prime}$ flanking region of $R P K 1$ was amplified by PCR with primers PARPDV1 (5'-TACCCCCTGCAGGGTCTCTCTGAGGTCATCAG-3') and M13 reverse primer. The primer PARPDV1 contains a terminal PstI site. PCR was performed with Pfu DNA polymerase (Stratagene, La Jolla, CA, U.S.A.) for highfidelity DNA synthesis. The amplified fragment was digested with PstI and inserted into the PstI site of pGDAS3 to obtain the gene-replacement vector pGDRPK1.

\section{Genomic DNA blot analysis.}

Total DNA of $C$. lagenarium was isolated from mycelia by the method described previously (Takano et al. 1997a). DNA digestion, agarose gel fractionation, labeling of probes, and hybridization were performed according to the manufacturer's instructions and standard methods (Sambrook et al. 1989). DNA probes were labeled with $\left[\alpha{ }^{32} \mathrm{P}\right] \mathrm{dCTP}$ (Amersham Pharmacia Biotech, Little Chalfont, Buckinghamshire, U.K.) with the BcaBEST labeling kit (Takara). Hybridization was carried out with hybridization buffer $(50 \%$ [vol/vol] formamide, $5 \times \mathrm{SSC}[1 \times \mathrm{SSC}$ is $0.15 \mathrm{M} \mathrm{NaCl}$ plus $0.015 \mathrm{M}$ sodium citrate], $0.1 \%$ [wt/vol] SDS [sodium dodecyl sulfate], and 5\% [wt/vol] sodium dextran sulfate) at $42^{\circ} \mathrm{C}$ for $16 \mathrm{~h}$. The membrane was washed at $42^{\circ} \mathrm{C}$ with $1 \times \mathrm{SSC}$ once for $1 \mathrm{~min}$ and twice for $30 \mathrm{~min}$.

\section{Fungal transformation.}

To obtain protoplasts, hyphae of $C$. lagenarium wild-type strain 104-T were incubated for 3 days in $200 \mathrm{ml}$ of potato sucrose broth supplemented with yeast extract (PSY; liquid extract from $200 \mathrm{~g}$ of potato, $20 \mathrm{~g}$ of sucrose, and $2 \mathrm{~g}$ of yeast extract per liter). Mycelia were harvested by filtration and treated with enzyme solution containing $5 \mathrm{mg}$ per $\mathrm{ml}$ of Lysing Enzyme from Trichoderma harzianum (Sigma Chemical, St. Louis, MO, U.S.A.) in $1.2 \mathrm{M} \mathrm{MgSO}_{4}$ and $10 \mathrm{mM}$ $\mathrm{Na}_{2} \mathrm{HPO}_{4}$ for 3 to $4 \mathrm{~h}$ to release the protoplasts (Rodriguez and Yoder 1987). Transformations were performed according to the method described previously (Vollmer and Yanofsky 1986). Twenty microliters of plasmid DNA solution $(0.5 \mu \mathrm{g}$ per $\mu \mathrm{l})$ was added to $1 \mathrm{ml}$ of protoplast solution $\left(5 \times 10^{7}\right.$ protoplasts in STC solution (1 M sorbitol; $50 \mathrm{mM}$ Tris- $\mathrm{HCl}, \mathrm{pH}$ 8.0; and $50 \mathrm{mM} \mathrm{CaCl}_{2}$ ). The protoplast solution mixed with 
plasmid DNA was incubated for $30 \mathrm{~min}$ on ice. Subsequently, $10 \mathrm{ml}$ of PEG solution (60\% polyethylene glycol; $50 \mathrm{mM}$ Tris- $\mathrm{HCl}, \mathrm{pH} 8.0$; and $50 \mathrm{mM} \mathrm{CaCl} 2$ ) was added gradually to the protoplast solution, then incubated for $15 \mathrm{~min}$ at room temperature. After removal of the PEG solution by centrifugation, protoplasts were suspended in $1 \mathrm{ml}$ of STC solution and mixed with $9 \mathrm{ml}$ of regeneration agar medium (3.12\% PDA and $0.6 \mathrm{M}$ glucose). The mixture was poured onto selection plates for hygromycin-resistant transformants (3.9\% PDA, 0.6 M glucose, and $100 \mu \mathrm{g}$ of hygromycin B per ml [Wako Pure Chemicals, Osaka, Japan]). Colonies growing on selection plates were selected on PDA medium containing $100 \mu \mathrm{g}$ of hygromycin per $\mathrm{ml}$ and screened.

\section{Pathogenicity test.}

Inoculation assay on cucumber (Cucumis sativus L. cv. Suyo) was performed as described (Takano et al. 1997a). Twentymicroliter drops of conidial suspension $\left(5 \times 10^{5}\right.$ conidia per $\left.\mathrm{ml}\right)$ were spotted on the surface of the cucumber leaves. After inoculation, the leaves were placed in humid petri dishes and incubated at $25^{\circ} \mathrm{C}$ for 7 days. When the leaves were inoculated through wounded sites, conidial suspension was spotted on the wounded sites ( 1 to $2 \mathrm{~mm}^{2}$ ) by scratching with $26 \mathrm{G}_{1 / 2}$ needles.

\section{Microscopy.}

To investigate appressorium formation and penetration of tested strains, $20 \mu \mathrm{l}$ of conidial suspension (approximately $10^{5}$ conidia per $\mathrm{ml}$ ) of each strain was spotted on the lower epidermis of cucumber cotyledons. To study germination and appressorium formation, inoculated cotyledons were incubated at $25^{\circ} \mathrm{C}$ for $12 \mathrm{~h}$ and the epidermal layers of cotyledons were peeled and observed by microscopy (Axioskop; Carl Zeiss, Thornwood, NY, US.A.). To investigate penetration, inoculated cotyledons were incubated at $25^{\circ} \mathrm{C}$ for 4 days and epidermal layers were peeled, stained with lactophenol aniline blue, and observed by microscopy (Takano et al. 1997a). For germination and appressorium formation assay in vitro, the conidial suspensions of tested strains (approximately $10^{5}$ conidia per $\mathrm{ml}$ ) were placed in glass petri dishes and incubated at $24^{\circ} \mathrm{C}$. To observe dormant conidia formed on mycelial cultures, conidia were collected from cultures and subjected directly to observation by microscopy. Microscopic images were captured with a chilled CCD camera (Argus 50; Hamamatsu Photonics, Hamamatsu, Japan).
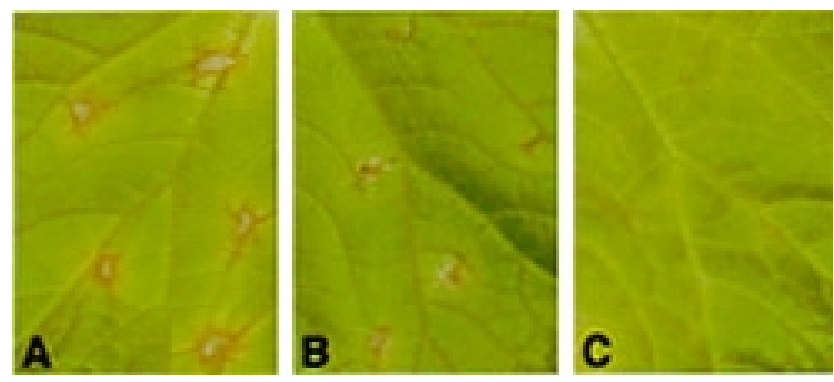

Fig. 7. Inoculation assay of wounded cucumber leaves. Detached cucumber leaves were wounded by scratching with a needle. Conidial suspensions of tested strains were spotted on the wounded sites of leaves. Inoculated leaves were incubated at $25^{\circ} \mathrm{C}$ for 7 days. A, Albino mutant 79215 ( $p k s 1$ ) was used as a positive control. B, rpkl mutant DPK1. C, Suppressor mutant SGC1.

\section{PKA assays.}

Protein extracts were prepared from mycelia grown in PSY for 3 days at $25^{\circ} \mathrm{C}$. Protein was extracted, as described previously (Yang and Dickman 1997). Mycelia were harvested by filtration through sterile gauze. Approximately 500 to $600 \mathrm{mg}$ of mycelia was ground with $500 \mathrm{mg}$ of acid-washed glass beads of $106 \mu \mathrm{m}$ and finer (Sigma) in liquid nitrogen and $500 \mu \mathrm{l}$ of protein extraction buffer (5 mM EDTA and $50 \mathrm{mM}$ Tris$\mathrm{HCl}, \mathrm{pH} 7.5)$ containing protease inhibitors aprotinin $(25 \mu \mathrm{g}$ per $\mu \mathrm{l})$ (Sigma) and phenylmethylsulfonyl fluoride $(1 \mathrm{mM})$ were added. After incubation on ice for $20 \mathrm{~min}$, the mixture was centrifuged at $12,000 \mathrm{rpm}$ at $4^{\circ} \mathrm{C}$ for $20 \mathrm{~min}$. Protein concentration in collected supernatant was adjusted to $500 \mu \mathrm{g}$ per $\mathrm{ml}$ by the method used by Bladford (1976) with bovine serum albumin as a standard. Five microliters of the adjusted samples were subjected to measurement of PKA activity. PKA activity assay was performed with fluorescent PKA model substrate Kemptide according to the manufacturer's instruction. The phosphorylated Kemptide, separated on an $0.8 \%$ agarose gel, was visualized by UV light, and the images were captured with a digital camera. The relative amount of each was determined with the National Institutes of Health image program, version 1.61 (Bethesda, MD, U.S.A.).

\section{ACKNOWLEDGMENTS}

We thank K. Mise and I. Furusawa for their valuable suggestions and support during the course of this work. We also thank T. K. Mitchell and R. A. Dean for critical reading of the manuscript. This work was supported, in part, by research grants from the Ministry of Education, Science, Sports and Culture of Japan.

\section{LITERATURE CITED}

Adachi, K., and Hamer, J. E. 1998. Divergent cAMP signaling pathways regulate growth and pathogenesis in the rice blast fungus Magnaporthe grisea. Plant Cell 10:1361-1373.

Agrios, G. N. 1988. Plant Pathology, 3rd ed. Academic Press, San Diego.

Bechinger, C., Giebel, K. F., Schnell, M., Leiderer, P., Deising, H. B., and Bastmeyer, M. 1999. Optical measurements of invasive forces exerted by appressoria of a plant pathogenic fungus. Science 285:18961899.

Beckerman, J. L., and Ebbole, D. J. 1996. MPG1, a gene encoding a fungal hydrophobin of Magnaporthe grisea, is involved in surface recognition. Mol. Plant-Microbe Interact. 9:450-456.

Bradford, M. M. 1976. A rapid and sensitive method for the quantitation of microgram quantities of protein utilizing the principle of proteindye binding. Anal. Biochem. 72:249-254.

Bruno, K. S., Aramayo, R., Minke, P. F., Metzenberg, R. L., and Plamann, M. 1996. Loss of growth polarity and mislocalization of septa in a Neurospora mutant altered in the regulatory subunit of cAMP-dependent protein kinase. EMBO. J. 15:5772-5782.

Cameron, S., Levin, L., Zoller, M., and Wigler, M. 1988. cAMP-independent control of sporulation, glycogen metabolism, and heat shock resistance in $S$. cerevisiae. Cell 53:555-566.

Choi, W., and Dean, R. A. 1997. The adenylate cyclase gene MAC1 of Magnaporthe grisea controls appressorium formation and other aspects of growth and development. Plant Cell 9:1973-1983.

de Jong, J. C., McCormack, B. J., Smirnoff, N., and Talbot, N. J 1997. Glycerol generates turgor in rice blast. Nature 389:244-245.

Gold, S. E., Duncan, G., Barrett, K., and Kronstad, J. W. 1994. cAMP regulates morphogenesis in the fungal pathogen Ustilago maydis. Genes Dev. 8:2805-2816.

Gold, S. E., Brogdon, S. M., Mayorga, M. E., and Kronstad, J. W. 1997. The Ustilago maydis regulatory subunit of a cAMP-dependent protein kinase is required for gall formation in maize. Plant Cell 9:1585-1594. Howard, R. J., Ferrari, M. A., Roach, D. H., and Money, N. P. 1991. 
Penetration of hard substrates by a fungus employing enormous turgor pressures. Proc. Natl. Acad. Sci. USA 88:11281-11284.

Inagaki, A., Takano, Y., Kubo, Y., Mise, M., and Furusawa, I. 2000. Construction of an equalized cDNA library from Colletotrichum lagenarium and its application to the isolation of differentially expressed genes. Can. J. Microbiol. 46:1-9.

Kim, Y.-K., Kawano, T., Li, D., and Kolattukudy, P. E. 2000. A mitogenactivated protein kinase kinase required for induction of cytokinesis and appressorium formation by host signals in the conidia of Colletotrichum gloeosporioides. Plant Cell 12:1331-1343.

Kronstad, J. W. 1997. Virulence and cAMP in smuts, blasts and blights. Trends Plant Sci. 2:193-199.

Kronstad, J. W, Maria, A. D., Funnell, D., Laidlaw, R. D., Lee, N., Moniz de Sa, M., and Ramesh, M. 1998. Signaling via cAMP in fungi: Interconnections with mitogen-activated protein kinase pathways. Arch. Microbiol. 170:395-404.

Kubo, Y., and Furusawa, I. 1991. Melanin biosynthesis: Prerequisite for successful invasion of the plant host by appressoria of Colletotrichum and Pyricularia. Pages 205-218 in: The Fungal Spore and Disease Initiation in Plants and Animals. G. T. Cole and H. C. Hoch, eds. Plenum Publishing, New York.

Kubo, Y., Takano, Y., Endo, N., Yasuda, N., Tajima, S., and Furusawa, I. 1996. Cloning and structural analysis of the melanin biosynthesis gene SCD1 encoding scytalone dehydratase in Colletotrichum lagenarium. Appl. Environ. Microbiol. 62:4340-4344.

Mitchell, T. K., and Dean, R. A. 1995. The cAMP-dependent protein kinase catalytic subunit is required for appressorium formation and pathogenesis by the rice blast pathogen Magnaporthe grisea. Plant Cell 7:1869-1878.

Pan, X., and Heitman, J. 1999. Cyclic AMP-dependent protein kinase regulates pseudohyphal differentiation in Saccharomyces cerevisiae. Mol. Cell Biol. 7:4874-4887.

Perpetua, N. S., Kubo, Y., Yasuda, N., Takano, Y., and Furusawa, I. 1996. Cloning and characterization of a melanin biosynthetic THRI reductase gene essential for appressorial penetration of Colletotrichum lagenarium. Mol. Plant-Microbe Interact. 9:323-329.

Podila, G. K., Rogers, L. M., and Kolattukudy, P. E. 1993. Chemical signals from avocado surface wax trigger germination and appressorium formation in Colletotrichum gloeosporioides. Plant Physiol. 103:267272.

Robertson, L. S, and Fink, G. R. 1998. The three yeast A kinases have specific signaling functions in pseudohyphal growth. Proc. Natl. Acad. Sci. USA 95:13783-13787.

Rodriguez, R. J., and Yoder, O. C. 1987. Selectable genes for transformation of the fungal plant pathogen Glomerella cingulata f. sp. phaseoli (Colletotrichum lindemuthianum). Gene 54:73-81.

Sambrook, J., Fritsch, E. F., and Maniatis, T. 1989. Molecular Cloning: A Laboratory Manual. 2nd ed. Cold Spring Harbor Laboratory, Cold Spring Harbor, NY, U.S.A.

Sweigard, J., Chumley, F., Carroll, A., Farrall, L., and Valent, B. 1997. A series of vectors for fungal transformation. Fungal Genet. Newsl. 44:52-55.

Takano, Y., Kubo, Y., Shimizu, K., Mise, K., Okuno, T., and Furusawa, I. 1995. Structural analysis of PKS1, a polyketide synthase gene involved in melanin biosynthesis in Colletotrichum lagenarium. Mol. Gen. Genet. 249:162-167.
Takano, Y., Kubo, Y., Kawamura, C., Tsuge, T., and Furusawa, I. 1997a. The Alternaria alternata melanin biosynthesis gene restores appressorial melanization and penetration of cellulose membranes in the melanin-deficient albino mutant of Colletotrichum lagenarium. Fungal Genet. Biol. 21:131-140.

Takano, Y., Kubo, Y., Kuroda, I., and Furusawa, I. 1997b. Temporal transcriptional pattern of three melanin biosynthesis genes, PKS1, SCD1, and THR1, in appressorium-differentiating and nondifferentiating conidia of Colletotrichum lagenarium. Appl. Environ. Microbiol. 63:351-354.

Takano, Y., Kikuchi, T., Kubo, Y., Hamer, J. E., Mise, K., and Furusawa, I. 2000. The Colletotrichum lagenarium MAP kinase gene CMK1 regulates diverse aspects of fungal pathogenesis. Mol. Plant-Microbe Interact. 13:374-383.

Taylor, S. S., Buechler, J. A., and Yonemoto, W. 1990. cAMP-dependent protein kinase: Framework for a diverse family of regulatory enzymes. Annu. Rev. Biochem. 59:971-1005.

Thines, E., Weber, R. W., and Talbot, N. J. 2000. MAP kinase and protein kinase A-dependent mobilization of triacylglycerol and glycogen during appressorium turgor generation by Magnaporthe grisea. Plant Cell 12:1703-1718.

Thompson, J. D., Higgins, D. G., and Gibson, T. J. 1994. CLUSTAL W: Improving the sensitivity of progressive multiple sequence alignment through sequence weighting, position-specific gap penalties and weight matrix choice. Nucleic Acids Res. 22:4673-4680.

Toda, T., Cameron, S., Sass, P., Zoller, M., Scott, J. D., McMullen, B., Hurwitz, M., Krebs, E. G., and Wigler, M. 1987. Cloning and characterization of $B C Y 1$, a locus encoding a regulatory subunit of the cyclic AMP-dependent protein kinase in Saccharomyces cerevisiae. Mol. Cell. Biol. 7:1311-1377.

Tokiwa, G., Tyers, M., Volpe, T., and Futcher, B. 1994. Inhibition of G1 cyclin activity by the Ras/cAMP pathway in yeast. Nature 371:342345 .

Vollmer, S. J., and Yanofsky, C. 1986. Efficient cloning of genes of Neurospora crassa Proc. Natl. Acad. Sci. USA 83:4869-4873.

Xu, J.-R., and Hamer, J. E. 1996. MAP kinase and cAMP signaling regulate infection structure formation and pathogenic growth in the rice blast fungus Magnaporthe grisea. Genes Dev. 10:2696-2706.

Xu, J.-R., Urban, M., Sweigard, J. A., and Hamer, J. E. 1997. The CPKA gene of Magnaporthe grisea is essential for appressorial penetration. Mol. Plant-Microbe Interact. 10:187-194.

Xu, J.-R., Staiger, C. J., and Hamer, J. E. 1998. Inactivation of the mitogen-activated protein kinase Mps1 from the rice blast fungus prevents penetration of host cells but allows activation of plant defense responses. Proc. Natl. Acad. Sci. USA 95:12713-12718.

Yang, Z., and Dickman, M. B. 1997. Regulation of cAMP and cAMP dependent protein kinase during conidial germination and appressorium formation in Colletotrichum trifolii. Physiol. Mol. Plant Pathol. 50: 117-127.

Yang, Z., and Dickman, M. B. 1999a. Colletotrichum trifolii mutants disrupted in the catalytic subunit of cAMP-dependent protein kinase are nonpathogenic. Mol. Plant-Microbe Interact. 12:430-439.

Yang, Z., and Dickman, M. B. 1999b. Molecular cloning and characterization of $C t-P K A R$, a gene encoding the regulatory subunit of cAMPdependent protein kinase in Colletotrichum trifolii. Arch. Microbiol. $171: 249-256$ 